framework which would incorporate employees' health factors into the risk assessment process which employers are familiar with.

Methods A literature review was conducted to gather the evidence for the association between chronic diseases and risk of injuries in the workplace. A health-based risk assessment framework was constructed that incorporates employees' health factors, giving examples on how this can be done, as well as highlighting some of the challenges.

Results Many recent studies found that increased body mass index was associated with higher risk of injuries, while older workers were at risk of more severe injuries. Diabetes mellitus was also associated with workplace injuries in some studies. There were fewer studies looking at other chronic diseases such as hypertension. We will share our health-based risk assessment framework incorporating employees' health into the risk assessment process, in a comparable way as that for occupational health and safety hazards.

Discussion Including a health-based risk assessment framework into the Risk Assessment matrix can improve the integration of workplace health and safety management.

\section{A FIELD TRIAL TO ASSESS THE EFFECTIVENESS OF SETTING UP MODEL OUTREACH STI/HIV CLINICS FOR FEMALE SEX WORKERS IN DELHI}

\footnotetext{
${ }^{1,2}$ Jitendra Kumar Meena* ${ }^{2}$ Suneela Garg. 'Geetanjali Medical College and Hospital, Udaipur, Rajasthan, India; ${ }^{2}$ Maulana Azad Medical College, New Delhi, India
}

\subsection{6/oemed-2018-ICOHabstracts. 1501}

Introduction Sexually transmitted infections (STI) are associated with increased risk of HIV infection and represent a major public health problem in developing countries. The UNAIDS global guidance on HIV and sex work (2010) recommends comprehensive, accessible, acceptable, user friendly STI/ HIV prevention and treatment based on local context and individual needs of female sex workers (FSWs).

Methods A field trial conducted to assess the effectiveness of specialised STI/HIV outreach clinic for FSWs. Pre and post intervention data were collected using semi-structured questionnaires (Cronbach's $a=0.74$ ) with health counselling (safe sex, condom promotion). Data obtained on demographic characteristics, knowledge, practices, morbidity profile and clinical treatment outcomes. The study done at sentinel STI/HIV clinic near to brothels for easy accessibility. Participant's baseline assessment and management done coupled with a counselling session. Participants were followed up after 7 days and 3 months and data analysed using Mc Nemars test.

Result Out of 200 participants 156 followed up in the study. The mean age was $28.6 \pm 5.2$ years and $58.2 \%$ were illiterate. $48.0 \%$ participant's reported pathological discharge suggestive of STI. On lab testing, 18 subjects were HIV seropositive and referred to ART centre. Partner management for STIs done in only $35.4 \%$ cases due to noncompliance. In pre-intervention phase majority $76.0 \%$ were aware of but only $41.0 \%$ knew transmission routes correctly. $78 \%$ had ever undergone HIV testing and only $36 \%$ used condoms regularly. Significant clinical improvement and behaviour change reported in $84.3 \%$ $(p=0.003)$ and $82.2 \%(p=0.01)$ cases respectively. There was an improved coverage, satisfaction for STI/HIV diagnostic and management services. The coverage of condom usage and voluntary HIV testing escalated to $71.0 \%(\mathrm{p} \leq 0.01)$ and $96.0 \%$ $(\mathrm{p}=0.034)$ respectively.

Discussion Creation of outreach clinics providing quality diagnostic and treatment aided by counselling services is an effective strategy for prevention and management of STI/HIV among high risk groups (FSWs).

\section{LIMITED FLUID AND RESTRICTED TOILETING ARE ASSOCIATED WITH REDUCED WORK PRODUCTIVITY IN WOMEN AT WORK}

${ }^{1}$ Heather Pierce*, ${ }^{1}$ Lin Perry, ${ }^{2}$ Robyn Gallagher, ${ }^{3}$ Pauline Chiarelli. ${ }^{1}$ University of Technology Sydney, Australia; ${ }^{2}$ Charles Perkins Centre, University of Sydney, Australia; ${ }^{3}$ University of Newcastle, Australia

\subsection{6/oemed-2018-ICOHabstracts. 1502}

Introduction Occupational demands influence workers' fluid intake and toileting behaviours. Access to fluids and toilets is necessary for bladder health and effective management of storage urinary symtoms, including urinary frequency, urgency and incontinence. These conditions are prevalent in women, yet little is known of the relationship between fluid intake, toileting and work productivity for those with storage urinary symptoms at work.

Methods A survey was distributed to 600 female nurses and midwives at three urban hospitals between June and November 2016. Logistic modelling was used to estimate the predictive association between limited fluid intake, restricted toileting and work impairment for those with storage urinary symptoms at work. A modified version of the Work Limitations Questionaire was used to measure time management, mental concentration and physical demands. We adjusted the model for age, body mass index, back pain, anxiety, depression, storage urinary symptoms at any time (not just at work) and other pelvic floor dysfunctions.

Results Of the included sample ( $\mathrm{n}=353), 77.1 \%$ (95\% CI: 73 to 81 ) delayed voiding at work, $22.4 \%$ (95\% CI: 18 to 27 ) could not access the toilet when required, and 26.9\% (95\% CI: 22 to 32 ) reduced fluid intake to delay or avoid voiding at work. Almost half of the sample $(46.7 \%$; 95\% CI: 42 to 52) reported storage urinary symptoms at work: those who limited fluids were more likely to have time management work impairment than those who didn't (OR: 3.87; 95\% CI: 1.63 to 9.15$)$. Those who delayed voiding were more likely to have concentration impairment than those who didn't (OR: 8.38; 95\% CI: 1.03 to 68.45 ).

Discussion A worker's capacity to access fluids and toilets at work is modifiable, hence associated urinary symptoms and productivity loss may be preventable. Occupation-specific barriers to healthy bladder behaviours should be identified and redressed. Bladder health promotion should be considered for inclusion in occupational health programs.

\section{WHY DO FEMALE WORKERS HAVE A GREATER RISK OF} LONG-TERM CHRONIC DISEASE COMPARED TO MEN?

\footnotetext{
${ }^{1}$ Allard E Dembe*, ${ }^{2}$ Xiaoxi Yao. ${ }^{1}$ The Ohio State University, College of Public Health, Columbus, Ohio, USA; ${ }^{2}$ Mayo Clinic, Robert D. and Patricia E. Kern Centre for the Science of Health Care Delivery, Rochester, Minnesota, U.S.A
}

10.1136/oemed-2018-ICOHabstracts. 1503 
Introduction Recent studies suggest that female workers face a greater risk of long-term chronic disease than male workers. A 2008 Mexican study indicated that older female workers have an elevated prevalence of arthritis, diabetes, and hypertension compared to men. A recent U.S. study showed that women working long hours have an elevated risk for chronic disease later in life compared to men. South Korean researchers reported that women in nonstandard jobs are more likely to suffer chronic mental disorders. However, little is known about why female workers have a comparatively greater risk of long term chronic disease.

Methods This study involves conducting a systematic literature review followed by a meta analysis of studies involving chronic disease among working women, particularly focusing on populations of working women who are aged fifty and older. The review also focuses on identifying distinctive aspects of women's work in specific occupational groups in an international context.

Result Initial results from the analysis suggest several explanations: a) the evidence is relatively strong that working long hours for extended periods of time raises the likelihood for chronic disease, b) the literature suggests that shift work and disruption of circadian rhythms among women can create long-term chronic health problems, c) women are often required to perform multiple roles at home and work which place greater stress on the women's ability to meet those additional responsibilities, d) because of those obligations, many working women may not have sufficient time to take care of their health, and e) some demanding physical tasks might be difficult to perform because of inherent gender-based biological constraints (e.g., performing heavy materials-handling tasks).

Discussion More specific empirical study of the reasons for elevated chronic disease risk among working women is needed. Additionally, workplace-based interventions to screen affected women for chronic disease should be adopted.

\section{THE MEDIATING ROLE OF RECOVERY OPPORTUNITIES ON FUTURE SICKNESS ABSENCE FROM A GENDER- AND AGE-SENSITIVE PERSPECTIVE}

${ }^{1} \mathrm{JS}$ Boschman, ${ }^{2} \mathrm{~A}$ Noor, ${ }^{1} \mathrm{JK}$ Sluiter, ${ }^{2} \mathrm{M}$ Hagberg* ${ }^{*}{ }^{1}$ Academic Medical Centre, University of Amsterdam, Department: Coronel Institute of Occupational Health, Amsterdam Public Health research institute, Amsterdam, The Netherlands; ${ }^{2}$ Occupational and Environmental Medicine, Sahlgrenska Academy and University Hospital, University of Gothenburg, Gothenburg, Sweden

\subsection{6/oemed-2018-ICOHabstracts.1504}

A lack of sufficient recovery during and after work may help to explain impaired health in the long run. We aimed to increase knowledge on the mediating role of recovery opportunities (RO) during and after work on future sickness absence from a gender- and age-sensitive perspective. We used data on RO from a Swedish national survey in 2011 and linked these to sickness absence ( $>14$ days) two years later among the general working population $(n=7,649)$. Mediation of the relationship between gender and sickness absence by exposure to RO was studied through linear regression. We conducted separate analyses for RO during and after work and for three different age groups $(16-29 ; 30-49 ; 50-64)$. The sample consisted of 3563 men and 4086 women. Sickness absence was higher among the women than among the men (11 days vs 5 days, $\mathrm{p}<0.001)$. Men reported statistically significantly more positive on their RO than women. RO during ( $\left.\begin{array}{ll}3 & 0.3-1.8)\end{array}\right)$ and after work ( $B$ 0.4-0.6) mediated the relationship between gender and sickness absence. Mediation effects existed across age groups, with the strongest effects of RO during work found among the age group between 50 and 64 years of age (attenuation 36\%). Our results indicate that gender inequality is also reflected in worse RO among women. This partially explains the increased risk of future sickness absence, particularly among those above 50 years of age. These findings show that RO during work deserve more attention in working life research.

\section{URINARY INCONTINENCE AND INTENTION TO LEAVE CURRENT JOB}

${ }^{1}$ Heather Pierce*, ${ }^{1}$ Lin Perry, ${ }^{2}$ Robyn Gallagher, ${ }^{3}$ Pauline Chiarelli. ${ }^{1}$ University of Technology Sydney, Australia; ${ }^{2}$ Charles Perkins Centre, University of Sydney, Australia; ${ }^{3}$ University of Newcastle, Australia

\subsection{6/oemed-2018-ICOHabstracts. 1505}

Introduction Urinary incontinence (UI) is a condition prevalent in women, with negative impact on pyschosocial health and quality of life. The aim of this study was to examine the relationship between urinary incontinence and work.

Methods An electronic survey was distributed between May 2014 and February 2015 to members of the New South Wales Nurses and Midwives Association in Australia. Urinary incontinence prevalence and severity was investigated using the International Consultation on Incontinence Questionnaire Urinary Incontinence - Short Form. Demographic, general and female reproductive health data known to influence the reporting of UI were collected. Investigated work characteristics included: work role, contract, shift, job satisfaction and plans to leave current job. Data analyses included female respondants and excluded those with a curent pregnancy or urinary tract infection 'sometimes or often' in the last 12 months.

Results The final sample size was 2,907, of mean age 47.35 years (19-74 \pm 11.58$)$; mean Body Mass Index $28.09 \mathrm{~kg} / \mathrm{m} 2$ $(15-57 \pm 6.41) ; 69.9 \%$ were parous. The prevalence of any UI in the past 4 weeks was $32.0 \%$ (95\% CI: $30 \%$ to $34 \%$; $\mathrm{n}=930$ ). Slight severity UI was present in $55.2 \%$, moderate UI in $40.5 \%$ and severe UI in $4.4 \%$. Those with severe UI were more dissatisfied with their work $(\mathrm{p}=0.001)$ and more likely to intend to leave their current position in the next 12 months than those with slight or moderate symptoms (OR: 2.26; 95\% CI: 1.02 to 5.01) after accounting for age, Body Mass Index, parity, anxiety, depression, work contract, shift and job satisfaction.

Conclusion UI is a health concern for women in the workforce, associated with reduced job satisfaction and an intention to leave current job. The findings of this study flag the need to raise the awareness of this common condition in the workplace as UI is a treatable and preventable condition.

\section{GENDER EQUITY IN EDITORIAL BOARDS OF MEDLINE INDEXED OCCUPATIONAL SAFETY \& HEALTH JOURNALS}

${ }^{1}$ Priyanka Roy*, ${ }^{2}$ Soumyadeep Bhaumik. 'Department of Labour, Government of West Bengal, India; ${ }^{2}$ Public Health Foundation of India, India

10.1136/oemed-2018-ICOHabstracts. 1506 